\title{
Saure Phosphatase im Serum (Substrat: $\alpha$-Naphthylphosphat): Referenzwerte und diagnostische Aussage
}

\author{
Von D. Seiler, D. Nagel \\ Städtische Krankenanstalten, Institut für Klinische Chemie, Ludwigshafen/Rh.
}

\section{W. Tritschler und S. Looser}

Boehringer Mannheim GmbH, Erprobung Diagnostica, Mannheim und Boehringer Mannheim GmbH, Biochemica Werk Tutzing, Tutzing

(Eingegangen am 20. September 1982/18. April 1983)

Zusammenfassung: Für eine modifizierte Methode nach Hillmann (-1) zur Bestimmung der katalytischen Konzentration an saurer Phosphatase im Serum (Substrat: $\alpha$-Naphthylphosphat) werden Ergebnisse mitgeteilt.

In einem Kollektiv von 158 Männern im Alter von 16 bis 85 Jahren ergab sich als Obergrenze des Referenzbereiches (95. Perzentil) für die gesamte saure Phosphatase $4,7 \mathrm{U} / 1\left(37^{\circ} \mathrm{C}\right)$ bzw. $4,2 \mathrm{U} / 1\left(30^{\circ} \mathrm{C}\right)$ und für die tartrathemmbare saure Phosphatase $1,6 \mathrm{U} / \mathrm{l}\left(37^{\circ} \mathrm{C}\right) \mathrm{bzw} .1,5 \mathrm{U} / \mathrm{l}\left(30^{\circ} \mathrm{C}\right)$. Bei 60 Frauen im Alter von 18 bis 80 Jahren lag die Obergrenze des Referenzbereiches (95. Perzentil) für die gesamte saure Phosphatase bei $3,7 \mathrm{U} / \mathrm{l}\left(37^{\circ} \mathrm{C}\right)$ bzw. $3,0 \mathrm{U} / 1\left(30^{\circ} \mathrm{C}\right)$.

Eine Altersabhängigkeit der katalytischen Konzentrationen war weder bei Männern noch Frauen nachweisbar.

Bei 89 Patienten mit Prostata-Carcinom der Stadien C und D wurde die katalytische Konzentration der tartrathemmbaren sauren Phosphatase mit den Substraten $\alpha$-Naphthylphosphat und $p$-Nitrophenylphosphat bestimmit.

Bei 74 dieser Patienten wurde die Konzentration an prostataspezifischer saurer Phosphatase mit einem Enzym-Immunoassay sowie einem Radio-Immunoassay ermittelt.

Die Sensitivität der Methode mit $p$-Nitrophenylphosphat ist unbefriedigend (66\%), die anderen Methoden ergeben eine bessere und vergleichbare Señsitivität (ca. 90\%). Die Ergebnisse mit $p$-Nitrophenylphosphat unterscheiden sich signifikant von denen mit $\alpha$-Naphthylphosphat als Substrat; die beiden immunchemischen Verfahren zeigen jedoch gegenüber der neuen Methode keinen signifikanten Unterschied.

Ės wird deshalb vorgeschlagen, die ältere Methode mit $p$-Nitrophenylphosphat zugunsten der neuen Methode aufzugeben. Katalytische Konzentrationen an tartrathemmbarer saurer Phosphatase im Grenzbereich normal-pathologisch $\left(1,6-2,0 \mathrm{U} / 1,37^{\circ} \mathrm{C}\right)$ sollten mit einem immunchemischen Verfahren kontrolliert werden.

Acid phosphatase in serum (substrate: $\alpha$-naphthylphosphate):

Reference values and diagnostic significance

Summary: We report the results obtained with a modification of Hillmann's method (1) for determination of the catalytic concentration of acid phosphatase in serum using $\alpha$-naphthylphosphate as the substrate. In a group of 158 males aged 16-85 years, the upper limit of the reference range ( 95 th percentile) for the total 
acid phosphatase was established as $4.7 \mathrm{U} / 1\left(37^{\circ} \mathrm{C}\right)$ and $4.2 \mathrm{U} / 1\left(30^{\circ} \mathrm{C}\right)$, respectively; the corresponding values for the tartrate-inhibited acid phosphatase were $1.6 \mathrm{U} / 1\left(37^{\circ} \mathrm{C}\right)$ and $1.5 \mathrm{U} / 1\left(30^{\circ} \mathrm{C}\right)$.

The upper limit of the reference range (95th percentile) for the total acid phosphatase determined in 60 females aged $18-80$ years was $3.7 \mathrm{U} / \mathrm{l}\left(37^{\circ} \mathrm{C}\right)$ and $3.0 \mathrm{U} / 1\left(30^{\circ} \mathrm{C}\right)$. The catalytic concentrations in men and women did not show any age-related differences.

The catalytic concentration of the tartrate-inhibited acid phosphatase was determined with the substrates $\alpha$ naphthylphosphate and $p$-nitrophenylphosphate in a group of 89 patients with prostatic carcinoma (stages $\mathrm{C}$ and D).

In 74 of these patients, the concentration of the prostatic specific acid phosphatase was assayed by enzymeimmunoassay and radioimmunoassay.

The sensitivity of the method with $p$-nitrophenylphosphate was found to be unsatisfactory $(66 \%)$, while that obtained with the other methods was superior and intercomparable (approx. $90 \%$ ). The results obtained with the two substrates ( $p$-nitrophenylphosphate vs. $\alpha$-naphthylphosphate) differed significantly. In contrast, the results given by the two immunochemical procedures did not exhibit a significant difference when compared with the new method.

In the light of the above, it is proposed that the previous method using $p$-nitrophenylphosphate be abandoned in favour of the new method. Catalytic concentrations of tartrate-inhibited acid phosphatase in the borderline range between normal and pathologic $\left(1.6-2.0 \mathrm{U} / 1,37^{\circ} \mathrm{C}\right)$ should be checked with an immunochemical method.

\section{Einführung}

Zur Bestimmung der katalytischen Aktivität der sauren Phosphatase im Serum (EC 3.1.3.2) werden verschiedene Substrate wie Thymolphthaleinphosphat, $p$-Nitrophenylphosphat oder $\alpha$-Naphthylphosphat eingesetzt. Für kein bisher bekanntes Substrat ist - auch nach Tartrathemmung - eine Spezifität für die prostataspezifische saure Phosphatase gegeben, obwohl Unterschiede bestehen (2). In Deutschland wird vorwiegend die Methode mit $p$-Nitrophenylphosphat eingesetzt.

Wir haben den von Hillmann (1) beschriebenen Test mit $\alpha$-Naphthylphosphat als Substrat modifiziert und untersucht, wie dieser Test von seiner klinischen Aussage her einzuordnen ist. Es wurden deshalb Referenzwerte ermittelt und bei Patienten mit Prostatacarcinom die katalytischen Konzentrationen an tartrathemmbarer saurer Phosphatase bestimmt. Die Ergebnisse wurden mit denen des Verfahrens von Fishman \& Lerner ((3) Substrat: p-Nitrophenylphosphat), einer enzymimmunologischen (4) und einer radioimmunologischen Methode (5) verglichen.

\section{Material und Methoden \\ Testprinzip}

Der Test zur Bestimmung der katalytischen Aktivität der sauren Phosphatase mit $\alpha$-Naphthylphosphat als Substrat wurde von Hillmann beschrieben (1) und in Bezug auf Fast Red TR- und Tartrat-Konzentration modifiziert (Monotest ${ }^{\circ}$ Saure Phosphata- se, Boehr̂inger Mannheim GmbH). Das bei der enzymatischen Hydrolyse von $\alpha$-Naphthylphosphat freigesetzte $\alpha$-Naphthol wird durch Kupplung mit diażotiertem 2-Amino-5-chlortoluol (Fast Red TR) zu einem Farbstoff ungigesetzt und dieser während der Reaktion ohne $\left(\Delta \mathrm{A}_{1} / \mathrm{min}\right)$ und mit Tartrat $\left(\Delta \mathrm{A}_{2} / \mathrm{min}\right)$ kontinuierlich bei $405 \mathrm{~nm}$ photometrisch gemessen.

\section{Endkonzentrationen im Test:}

Citrat-Puffer 0,2 mol/ $(\mathrm{pH} 5,2)$;

$\alpha$-Naphthylphosphat $10 \mathrm{mmol} /$,

Fast Red TR-Salz (diazotiertes 2-Amino-5-chlortoluol) 2,5 $\mathrm{mmol} / \mathrm{l}$;

Natriumtartrat $135 \mathrm{mmol} / \mathrm{h}$.

Berechnung: Gesamte saure Phosphatase:

$$
\begin{aligned}
& U / 1\left(30^{\circ} \mathrm{C}, 37^{\circ} \mathrm{C}\right)=743 \cdot \Delta A_{1} / \text { min } \\
& \text { Tartrathemmbare saure P̈hosphatase: } \\
& U / /\left(30^{\circ} \mathrm{C}, 37^{\circ} \mathrm{C}\right)=743 \cdot\left(\Delta \mathrm{A}_{1} / \mathrm{min}-\Delta \mathrm{A}_{2} / \mathrm{min}\right) \text {. }
\end{aligned}
$$

Dưrch Reduzierung der Fast Red TR-Konzentration auf 2;5 $\mathrm{mmol} / \mathrm{l}$ werden sowohl die Reageñzstabilität als auch die Lineani tät der Geschwiñdigkeit der enzymkatalysierten Reaktiōn inşbesondere bei $37^{\circ} \mathrm{C}$ Meßtemperatur verbessert. Die Tartrat-Konzentration von $135 \mathrm{mmol} / \mathrm{l}$ liegt deutlich im Hemm-Optimum. Im Gegensatz dazu ist die Tartrathemmung mit der im Original vorgeschlagenen Konzentration von $16 \mathrm{mmol} / /$ nicht vollständig. Der Pufferlösung ist als Konservierungsmittel Natriumbenzoàt zugesetzt. Die Reagenztabletten enthalten übliche Tablettierhilfsstof$\mathrm{fe}$, welche eine geringe Veränderung des Absorptionskoeffizienten bewirken $\left(\varepsilon_{405 \mathrm{~nm}}=148 \mathrm{~m}^{2} / \mathrm{mol} \triangleq 14,8 \cdot 10^{3} \mathrm{l} \cdot \mathrm{mol}^{-1} \cdot \mathrm{cm}^{-1}\right)$. Aktivatoren sind im Testansatz nicht enthalten.

\section{Testcharakteristik}

Wegen der geringen katalytischen Konzentration der sauren Phosphatase im Serum ist die Messung bei $30^{\circ} \mathrm{C}$ bžw. $37^{\circ} \mathrm{C}$ der bei $25^{\circ} \mathrm{C}$ vorzuziehen. Als Probenmateriāl wird Serum eingesetżt. Darüber hinaus kann nur noch Citratplasma verwendet werden, alle anderen üblichen Antikoagulantien führen zu Trübüngen im Testansatż. Die Verwendung von Citratplasma erfordert die Ermittlung gesonderter Referenżwerte, da im Serum durch Freiset- 
zung von z.T. ebenfalls tartrathemmbarer Thrombocyten-Phosphatase höhere Aktivitäten gemessen werden.

Serum kann ohne Stabilisierung und Aktivitätsverlust über mindestens 1 Monat bei $-20^{\circ} \mathrm{C}$ aufbewahrt werden. Lagerung bei $-4^{\circ} \mathrm{C}$ oder $15-25^{\circ} \mathrm{C}$ erfordert eine Stabilisierung des Serums z. B. durch Ansäuern mit $50 \mathrm{~g} /(0,8 \mathrm{~mol} / \mathrm{l})$ Essigsäure $(40 \mu \mathrm{l} / \mathrm{ml}$ Serum). Danach beträgt die Haltbarkeit drei Tage bzw. 24 Stunden. Die Testempfindlichkeit beträgt für $\Delta 0,001 \mathrm{~A} / \mathrm{min}=0,7$ U/. Der lineare Proportionalitätsbereich ist bei Einhaltung einer Vorinkubationszeit von $5 \mathrm{~min}$ und einer Meßzeit von $3 \mathrm{~min}$ bis zu einer Absorptionsdifferenz von $\Delta \mathbf{A} / \mathbf{m i n}=0,1$ gegeben, die einer katalytischen Konzentration von $74 \mathrm{U} / \mathrm{l}$ entspricht.

In Aufstockversuchen konnte zugesetzte Prostata-PhosphataseAktivität $\mathrm{zu} 100 \%$ wiedergefunden werden.

Aufgrund der Differenzmessungen ergeben sich für die tartrathemmbare saure Phosphatase deutlich schlechtere Präzisionen als für die gesamte saure Phosphatase. In vitro-Störungen durch Pharmaka konnten nicht festgestellt werden. Metabolite mit Ausnahme von Bilirubin stören den Test nicht. In Seren mit erhöhtem Gehalt an direktem Bilirubin werden zu niedrige katalytische Konzentrationen gemessen (6). Obwohl a-Naphthylphosphat von Erythrocyten-Phosphatase nur schlecht gespalten wird, ist hämolytisches Serum nicht geeignet. In Abhängigkeit von der Hämoglobinkonzentration werden zunächst zu tiefe, dann wieder ansteigende Aktivitäten gemessen.

So lange durch Einsatz lipämischer Seren die Ausgangsabsorption nicht zu hoch liegt und den gerätebedingten linearen Meßbereich nicht überschreitet, ist keine Beeinflussung des Tests gegeben.

\section{Methoden, Reagenzien, Geräte}

Die Untersuchungen erfolgten in Serum. Die Testpackungen zur Bestimmung von $L-\gamma$-Glutamyltransferase (EC 2.3.2.2), Aspartataminotransferase (EC 2.6.1.1), Alaninaminotransferase (EC 2.6.1.2), Lactatdehydrogenase (EC 1.1.1.27), alkalischer Phosphatase (EC 3.1.3.1) entstammten dem Lieferprogramm von Boehringer Mannheim GmbH, D-6800 Mannheim 31 (optimierte Methoden, $25^{\circ} \mathrm{C}$ ). In Tabelle 1 sind Angaben zu den übrigen Methoden bzw. zu den benutzten Geräten aufgeführt.

\section{Probanden für die Referenzwertermittlung}

Das Ausgangskollektiv der Männer $(\mathbf{N}=158)$ setzte sich überwiegend $(N=100)$ aus Blutspendern und Krankenhauspersonal, das arbeitsmedizinisch untersucht wurde, zusammen. Bei den restlichen 58 - besonders älteren - Probanden ( $>40$ Jahre) han- delt es sich um Patienten der Haut- und Augenklinik sowie der Hals-Nasen-Ohrenklinik. Die Einweisungsdiagnosen waren: Dermatitis, Asthma bronchiale, Katarakt, Keratitis, Otitis, Tonsillitis. Anamnese und rektale Untersuchung ergaben keinen Hinweis auf Erkrankungen der Prostata.

Das Ausgangskollektiv der Frauen $(\mathrm{N}=60)$ bestand überwiegend aus Personen, die arbeitsmedizinisch untersucht wurden. Schwangerschaft, erkennbare Erkrankungen sowie Therapie mit Medikamenten lagen nicht vor.

Zur Auswahl eines Referenzkollektivs wurde die katalytische Konzentration von Enzymen bestimmt, die bei Patienten mit Malignomen verändert sein können (7):

\section{Referenzwerte}

Tartrathemmbare saure Phosphatase (Substrat: $p$-Nitrophenylphosphat): Männer: bis $3 \mathrm{U} / 1\left(37^{\circ} \mathrm{C}\right)$;

gesamte saure Phosphatase (Substrat: $p$-Nitrophenylphosphat): Frauen: bis $11 \mathrm{U} / \mathrm{l}\left(37^{\circ} \mathrm{C}\right)$;

Aspartataminotransferase: Männer bis $18 \mathrm{U} / \mathrm{l}$, Frauen bis $15 \mathrm{U} / \mathrm{l}$ $\left(25^{\circ} \mathrm{C}\right)$;

Alaninaminotransferase: Männer bis $24 \mathrm{U} / 1$, Frauen bis $18 \mathrm{U} / \mathrm{l}$ $\left(25^{\circ} \mathrm{C}\right)$;

$L-\gamma$-Glutamyltransferase: Männer bis $28 \mathrm{U} / \mathrm{l}$, Frauen bis $18 \mathrm{U} / \mathrm{l}$ $\left(25^{\circ} \mathrm{C}\right)$;

Lactatdehydrogenase: bis $240 \mathrm{U} / 1\left(25^{\circ} \mathrm{C}\right)$;

alkalische Phosphatase: Jugendliche (16 Jahre): bis $700 \mathrm{U} / 1$ $\left(25^{\circ} \mathrm{C}\right)$; Erwachsene: 60 bis $185 \mathrm{U} / 1\left(25^{\circ} \mathrm{C}\right)$.

\section{Patienten mit Prostata-Carcinom}

Die Patienten waren 53 bis 88 Jahre alt. Die Diagnosen wurden durch rektale Palpation gestellt und durch Feinnadelbiopsie der Prostata gesichert. Die meisten Patienten hatten ein Carcinom im Stadium D, der geringere Teil ein Carcinom im Stadium C. Eine spezielle Therapie war vor der Einweisung nicht erfolgt.

\section{Probennahme und Qualitätssicherung}

Die Blutabnahme erfolgte vormittags. Nach Lösen der Stauung wurde Blut für die Serumgewinnung entnommen. Serum wurde nach $30 \mathrm{~min}$ Stehenlassen durch $10 \mathrm{~min}$ Zentrifugieren bei $2000 \mathrm{~g}$ erhalten. Das Serum wurde portioniert. In einem Teil wurden am selben Tag die katalytischen Konzentrationen von Enzymen bestimmt (vgl. Tab. 5; Ausnahme: saure Phosphatase). Für die immunchemischen Methoden wurde ein weiterer Teil bei $-20^{\circ} \mathrm{C}$ eingefroren und innerhalb von 1 Monat untersucht. Für die Be-

Tab. 1. Methoden, Reagenzien, Geräte und Meßbedingungen.

\begin{tabular}{|c|c|c|}
\hline Methoden und Reagenzien & Geräte & Messung \\
\hline $\begin{array}{l}\text { Hillmann ((1), modifiziert; EC 3.1.3.2 } \\
\text { Substrat: } \alpha \text {-Naphthylphosphat; monotest } \\
\text { Saure Phosphatase, } \\
\text { Boehringer Mannheim GmbH) }\end{array}$ & $\begin{array}{l}\text { Eppendorf } 1101 \mathrm{M} \text { mit Küvettenwechsel- } \\
\text { automatik, } \mathrm{Hg} 405 \mathrm{~nm}, 1 \mathrm{~cm} \text { Schichtdicke } \\
\text { (Netheler \& Hinz, D-2000 Hamburg) }\end{array}$ & $\begin{array}{l}30^{\circ} \mathrm{C}, 37^{\circ} \mathrm{C} \text { : Kontinuierlich, Einfach- } \\
\text { bestimmungen (Serum) }\end{array}$ \\
\hline $\begin{array}{l}\text { Fishman \& Lerner ((3); EC 3.1.3.2 } \\
\text { Substrat: } p \text {-Nitrophenylphosphat; } \\
\text { Testcombination Saure Phosphatase, } \\
\text { Boehringer Mannheim GmbH) }\end{array}$ & $\begin{array}{l}\text { Eppendorf } 1101 \mathrm{M} \text { mit } \\
\text { Absaugküvette (s. o.) }\end{array}$ & $\begin{array}{l}37^{\circ} \mathrm{C} \text { : Zweipunktmessung, Einfach- } \\
\text { bestimmungen (Serum) }\end{array}$ \\
\hline $\begin{array}{l}\text { Enzym-Immunoassay nach Grenner \& } \\
\text { Schmidtberger }(A)\end{array}$ & Eppendorf $1101 \mathrm{M}, \mathrm{Hg} 492 \mathrm{~nm}$ (s. o.) & Doppelansatz (Serum) \\
\hline Radio-Immunoassay nach Foti et al. (5) & $\begin{array}{l}\text { Rack-Gamma II, LKB-Instruments, } \\
\text { D-8032 Gräfelfing }\end{array}$ & Doppelansatz (Serum) \\
\hline
\end{tabular}


stimmung der katalytischen Konzentrationen an saurer Phosphatase wurde ein dritter Teil mit Essigsäure $(0,8 \mathrm{~mol} / \mathrm{l})$ versetzt (40 $\mu \mathrm{l} / \mathrm{ml}$ Serum). Die Verdünnung wurde bei der Berechnung vernachlässigt. Diese Seren wurden kühlgestellt $\left(4^{\circ} \mathrm{C}\right)$ und am selben Tag untersucht (Referenzwertermittlung) bzw. bei $-20^{\circ} \mathrm{C}$ eingefroren und innerhalb von 1 Monat untersucht (Untersuchungen bei Patienten).

Dic Präzision der Meßergebnisse für die saure Ṗhosphatase (Substrat: $\alpha$-Naphthylphosphat) wurde mit Kontrollseren (Precinorm ${ }^{\circledR}$ $\mathrm{U}$, Charge 09559, und Precipath ${ }^{\circledR} \mathrm{U}$, Charge 09510) sowie einem portioniert eingefrorenen laboreigenen Serumpool überprüft. Vor Beginn der Untersuchungen und nach Untersuchung von je 50 Seren wurden bei $30^{\circ} \mathrm{C}$ bzw. $37^{\circ} \mathrm{C}$ an 5 Tagen jeweils 6-fach-Bestimmungen durchgeführt.

Die Wiederfindung wurde mit Precinorm ${ }^{\circledR} \mathrm{U}$ und Precipath ${ }^{\circledR} \mathrm{U}$ (Chargen 1-570/1-513) überprüft, indem an jedem Untersuchungstag bei $30^{\circ} \mathrm{C}$ und $37^{\circ} \mathrm{C}$ jeweils Einfachbestimmungen durchgeführt wurden.

Die Qualitätssicherung für alle anderen Bestimmungen erfolgte durch mehrfache tägliche Kontrolle von Präzision und Richtigkeit der Methoden mit Kontrollseren.

\section{Statistische Methoden}

Die Präzisionen in der Serie und von Serie zu Serie wurden über die einfache Varianzanalyse berechnet. Die Verteilung der Meßwerte wurde durch Angabe der Perzentile beschrieben.

Auf unterschiedliche katalytische Konzentrationen der sauren Phosphatase in den einzelnen Altersdekaden wurde mit dem $\mathrm{H}-$ Test nach Kruskal-Wallis geprüft (8). Zur Úberprüfung eines Unterschieds zwischen Endkollektiv und aussortiertem Kollektiv bezüglich der katalyțischenen Konzentrationen der sauren Phosphatase wurde der U-Test von Mañn-Whitney eingesetzt (8). Der Vergleich verschiedener Methoden bei Patienten mit Prostata-Carcinom wurde mit dem von McNemar modifizierten Vorzeichentest durchgeführt (8). Als İrtumswahrscheinlichkeit würde jeweils $\alpha$ $=0,05$ festgelegt.

\section{Ergebnisse und Diskussion}

\section{Qualitätssicherung}

Die Tabellen 2 und 3 enthalten die Ergebnisșe der Qualitätssicherung in Kontrollseren und in einem

Tab. 2. Saure Phosphatase (Substrat: $\alpha$-Naphthylphosphat): Präzisionen in Kontrollseren und Poolserum.

\begin{tabular}{|c|c|c|c|c|c|}
\hline \multirow[t]{2}{*}{ Meßtemperatur $\left[{ }^{\circ} \mathrm{C}\right]$} & \multirow[t]{2}{*}{ Probenmaterial } & \multirow[t]{2}{*}{ Saure Phosphatase } & \multirow{2}{*}{$\begin{array}{l}\text { Katalyt. Konzentration } \\
\text { Mediạn } \\
{[\mathrm{U} / \mathrm{l}]}\end{array}$} & \multicolumn{2}{|c|}{ Variationskoeffizient } \\
\hline & & & & $\begin{array}{l}\text { Serie } \\
{[\%]}\end{array}$ & $\begin{array}{l}\text { Tag/Tag } \\
{[\%]}\end{array}$ \\
\hline \multirow{3}{*}{30} & Precinorm ${ }^{\circledast} \mathrm{U}$ & $\begin{array}{l}\text { gesamte } \\
\text { tartrathemmbare }\end{array}$ & $\begin{array}{l}8 ; 0 \\
3,7\end{array}$ & $\begin{array}{l}3,1 \\
5,3\end{array}$ & $\begin{array}{l}3,0 \\
5,7\end{array}$ \\
\hline & Precipath $^{\circledR} \mathrm{U}$ & $\begin{array}{l}\text { gesamte } \\
\text { tartrathemmbare }\end{array}$ & $\begin{array}{r}11,2 \\
5,5\end{array}$ & $\begin{array}{l}2,2 \\
4,3\end{array}$ & $\begin{array}{l}2,9 \\
5,0\end{array}$ \\
\hline & Poolserum & $\begin{array}{l}\text { gesamte } \\
\text { tartrathemmbare }\end{array}$ & $\begin{array}{l}3,5 \\
1,7\end{array}$ & $\begin{array}{l}5,1 \\
7,8\end{array}$ & $\begin{array}{l}5,1 \\
8,2\end{array}$ \\
\hline \multirow{3}{*}{37} & Precinorm ${ }^{\circledR} \mathrm{U}$ & $\begin{array}{l}\text { gesamte } \\
\text { tartrathemmbare }\end{array}$ & $\begin{array}{r}10,0 \\
4,5\end{array}$ & $\begin{array}{l}1,4 \\
5,6\end{array}$ & $\begin{array}{l}3,1 \\
8,8\end{array}$ \\
\hline & Precipath $^{\circledR} U$ & $\begin{array}{l}\text { gesamte } \\
\text { tartrathemmbare }\end{array}$ & $\begin{array}{r}14,6 \\
6,5\end{array}$ & $\begin{array}{l}1,4 \\
3,6\end{array}$ & $\begin{array}{l}2,2 \\
4,0\end{array}$ \\
\hline & Poolserum & $\begin{array}{l}\text { gesamte } \\
\text { tartrathemmbare }\end{array}$ & $\begin{array}{l}4,2 \\
2,2\end{array}$ & $\begin{array}{l}4,4 \\
8,3\end{array}$ & $\begin{array}{l}6,1 \\
9,2\end{array}$ \\
\hline
\end{tabular}

Tab. 3. Saure Phosphatase (Substrat: $\alpha$-Naphthylphosphat): Wiederfindung in Kontrollseren.

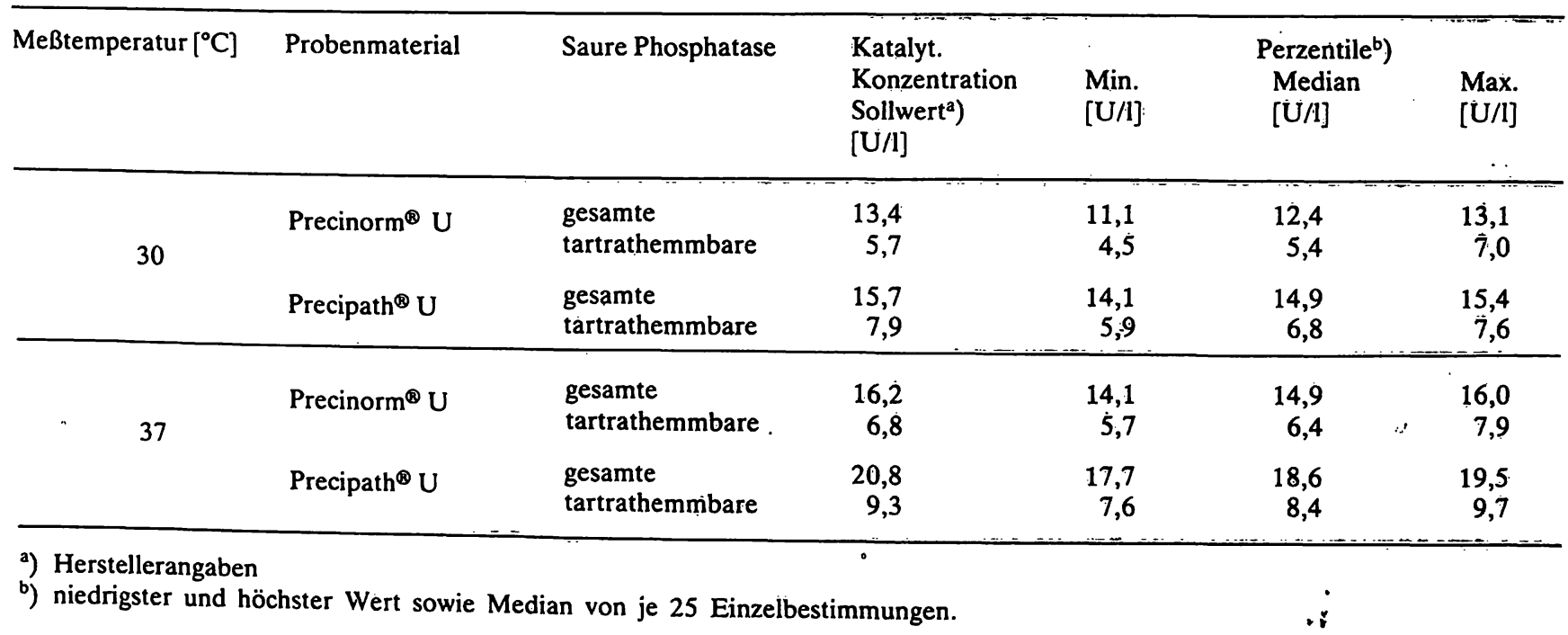


Poolserum. Die Präzisionen in der Serie bzw. von Tag zu Tag in Kontrollseren sind während des Untersuchungszeitraumes gut (gesamte saure Phosphatase: VK 1,4 bis $3,1 \%$ ) bzw. befriedigend (tartrathemmbare saure Phosphatase: VK 3,6 bis $8,8 \%$ ). Die Untersuchung des Poolserums ergibt Aufschlüsse über die Präzisionen im Entscheidungsbereich normal/pathologisch. Die Präzisionen für die gesamte saure Phosphatase (VK 4,4 bis 6,1\%) sowie die tartrathemmbare saure Phosphatase (VK 7,8 bis $9,2 \%$ ) sind noch akzeptabel.

Die Wiederfindungen in Kontrollseren (Tab. 3) sind befriedigend: Die Meßwerte liegen gut innerhalb des Sollwertbereiches, der gefundene Median liegt tiefer als der vom Hersteller angegebene Sollwert.

\section{Referenzkollektiv und Referenzwerte}

Tabelle 4 zeigt die Verteilung der katalytischen Konzentrationen an saurer Phosphatase im Ausgangskollektiv bei verschiedenen Temperaturen $\left(30^{\circ} \mathrm{C}, 37^{\circ} \mathrm{C}\right)$ für Männer und Frauen.

Für die tartrathemmbare saure Phosphatase stimmen (sowohl bei $30^{\circ} \mathrm{C}$ als auch $37^{\circ} \mathrm{C}$ Meßtempera- tur) das 95. und das 97,5. Perzentil praktisch überein.

Für die gesamte saure Phosphatase liegt das 97,5. Perzentil wesentlich höher als das 95. Perzentil (z.B. $37^{\circ} \mathrm{C}: 6,5 \mathrm{U} / 1$ gegenüber 4,7 U/1). Die Angabe des 97,5. Perzentils als Obergrenze des Referenzbereiches führt deshalb - sofern ausschließlich die gesamte saure Phosphatase bestimmt wird - für Diagnose und Therapie des Prostata-Carcinoms zu einer höheren Zahl falsch-negativer Ergebnisse. Wird dagegen das 95. Perzentil als Obergrenze gewählt, dann wird sich für die gesamte saure Phosphatase die Zahl der falsch-positiven Befunde erhöhen, d.h. die Spezifität des Testes wird sich erniedrigen. Diese verringerte Spezifität bei der Messung der Aktivität der gesamten sauren Phosphatase kann aber durch die gleichzeitige Bestimmung des tartrathemmbaren Anteils, der immer mitbestimmt werden sollte, verbessert werden. Die Obergrenze des Referenzbereiches wird deshalb auf das 95. Perzentil festgelegt.

Wie die Verteilungen der in Tabelle 5 dargestellten katalytischen Konzentrationen zeigen, enthält das Ausgangskollektiv einige Probanden, bei denen Erkrankungen nicht auszuschließen sind. Setzt man die

Tab. 4. Saure Phosphatase (Substrat: $\alpha$-Naphthylphosphat) im Serum: Verteilung der katalytischen Konzentrationen im Ausgangskollektiv.

Die Werte geben das 2,5., 5., 50. (Median), 95. und 97,5. Perzentil sowie niedrigste und höchste Werte (U/I) bei verschiedenen Temperaturen für $\mathbf{N}$ Probanden an.

\begin{tabular}{|c|c|c|c|c|c|c|c|c|c|c|}
\hline \multirow{2}{*}{$\begin{array}{c}\text { MeBtemperatur } \\
{\left[{ }^{\circ} \mathrm{C}\right]}\end{array}$} & \multirow[t]{2}{*}{ Probanden } & \multirow[t]{2}{*}{$\mathbf{N}$} & \multirow[t]{2}{*}{ Saure Phosphatase } & \multicolumn{7}{|c|}{ Katalytische Konzentration [U/I] } \\
\hline & & & & Min. & $2,5 \%$ & $5 \%$ & $50 \%$ & $95 \%$ & $97,5 \%$ & Max. \\
\hline \multirow{3}{*}{30} & \multirow{2}{*}{ Männer } & \multirow{2}{*}{158} & gesamte & 0,7 & 1,0 & 1,3 & 3,0 & 4,2 & 5,2 & 5,9 \\
\hline & & & tartrathemmbare & 0,3 & 0,4 & 0,4 & 0,7 & 1,5 & 1,5 & 1,7 \\
\hline & Frauen & 60 & gesamte & 0,7 & 0,7 & 1,3 & 1,9 & 3,0 & 3,0 & 3,2 \\
\hline \multirow{3}{*}{37} & \multirow{2}{*}{ Männer } & \multirow{2}{*}{158} & gesamte & 1,3 & 1,9 & 2,2 & 3,7 & 4,7 & 6,5 & 6,9 \\
\hline & & & tarțathemmbare & 0,4 & 0,7 & 0,7 & 1,2 & 1,6 & 1,7 & 2,0 \\
\hline & Frauen & 60 & gesamte & 1,3 & 1,5 & 2,0 & 2,8 & 3,7 & 3,7 & 3,7 \\
\hline
\end{tabular}

Tab. 5. Verteilung der katalytischen Konzentrationen von Enzymen im Serum von Probanden des Ausgangskollektivs. Die Werte geben das 2,5., 50. (Median) und 97,5. Perzentil sowie niedrigste und höchste Werte (U/I) von 218 Probanden an (158 Männer und 60 Frauen).

\begin{tabular}{|c|c|c|c|c|c|c|}
\hline \multirow[t]{2}{*}{ Enzym } & \multirow[t]{2}{*}{ Kollektiv } & \multicolumn{5}{|c|}{ Katalytische Konzentration [U/I] } \\
\hline & & Min. & $2,5 \%$ & $50 \%$ & $97,5 \%$ & Max. \\
\hline \multirow{2}{*}{$\begin{array}{c}\left.\text { Saure Phosphatase }{ }^{a}\right), \text { tartrathemmbare } \\
\text { gesamte }\end{array}$} & Männer & 0,5 & 0,6 & 1,3 & 2,5 & 3,0 \\
\hline & Frauen & 3,1 & 3,1 & 4,9 & 7,7 & 7,7 \\
\hline \multirow{5}{*}{$\begin{array}{l}\text { Aspartataminotransferase } \\
\text { Alaninaminotransferase } \\
\text { Lactatdehydrogenase } \\
\text { Alkalische Phosphatase } \\
L-\gamma-\text { Glutamyltransferase }\end{array}$} & Mạ̈nner & 5 & 6 & 10 & 18 & 21 \\
\hline & und & 6 & 7 & 12 & 25 & 27 \\
\hline & Frauen & 70 & 100 & 144 & 220 & 258 \\
\hline & & 10 & 60 & 105 & 320 & 670 \\
\hline & & 4 & 6 & 12 & 34 & 594 \\
\hline
\end{tabular}

a) Substrat: p-Nitrophenylphosphat. 
jeweiligen Referenzwerte als Ausschlußkriterien an, dann ergibt sich eine Reduzierung des Kollektivs um 31 auf 187. Zwischen dem Endkollektiv ( $N=187$ ) und dem aussortierten Kollektiv $(\mathrm{N}=31)$ besteht jedoch weder in Lage noch Verteilung der katalytischen Konzentrationen an saurer Phosphatase ein Unterschied.

Das Ausgangskollektiv wird deshalb zugleich als Referenzkollektiv angesehen.

Die Altersverteilung der katalytischen Konzentrationen an tartrathemmbarer saurer Phosphatase ist in Abbildung 1 für Männer angegeben: Eine Altersabhängigkeit der Werte wurde weder für Männer noch für Frauen (gesamte saure Phosphatase) gefunden.

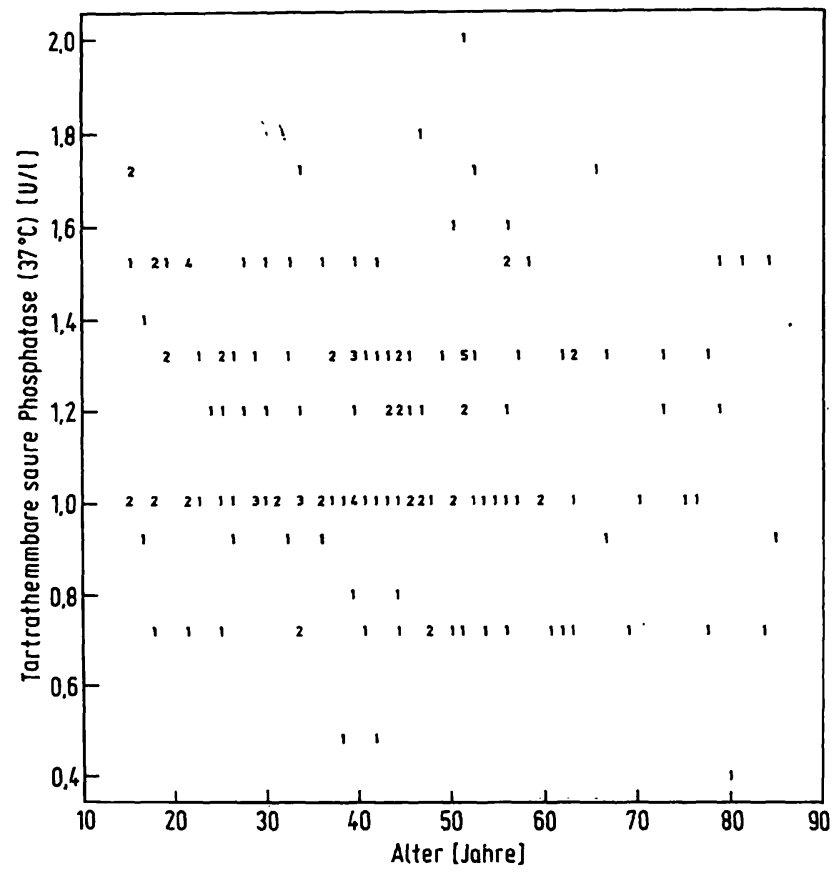

Abb. 1. Tartrathemmbare saure Phosphatase (Substrat: $\alpha$-Naphthylphosphat): Häufigkeitsverteilung der katalytischen Konzentrationen im Serum der Männer $(N=158)$ in Abhängigkeit vom Alter.

Wir schlagen deshalb folgende Referenzwerte vor: gesamte saure Phosphatase,

Männer: bis $4,7 \mathrm{U} / 1\left(37^{\circ} \mathrm{C}\right)$ bzw. $4,2 \mathrm{U} / 1\left(30^{\circ} \mathrm{C}\right)$; Frauen: bis $3,7 \mathrm{U} / 1\left(37^{\circ} \mathrm{C}\right)$ bzw. $3,0 \mathrm{U} / 1\left(30^{\circ} \mathrm{C}\right)$;

tartrathemmbare saure Phosphatase,

Männer: bis $1,6 \mathrm{U} / 1\left(37^{\circ} \mathrm{C}\right)$ bzw. $1,5 \mathrm{U} / 1\left(30^{\circ} \mathrm{C}\right)$.

\section{Diagnostische Aussage}

In Abbildung 2 ist die Verteilung der Meßwerte an saurer Phosphatase im Serum von Patienten mit Prostata-Carcinom (Stadien C und D) angegeben.

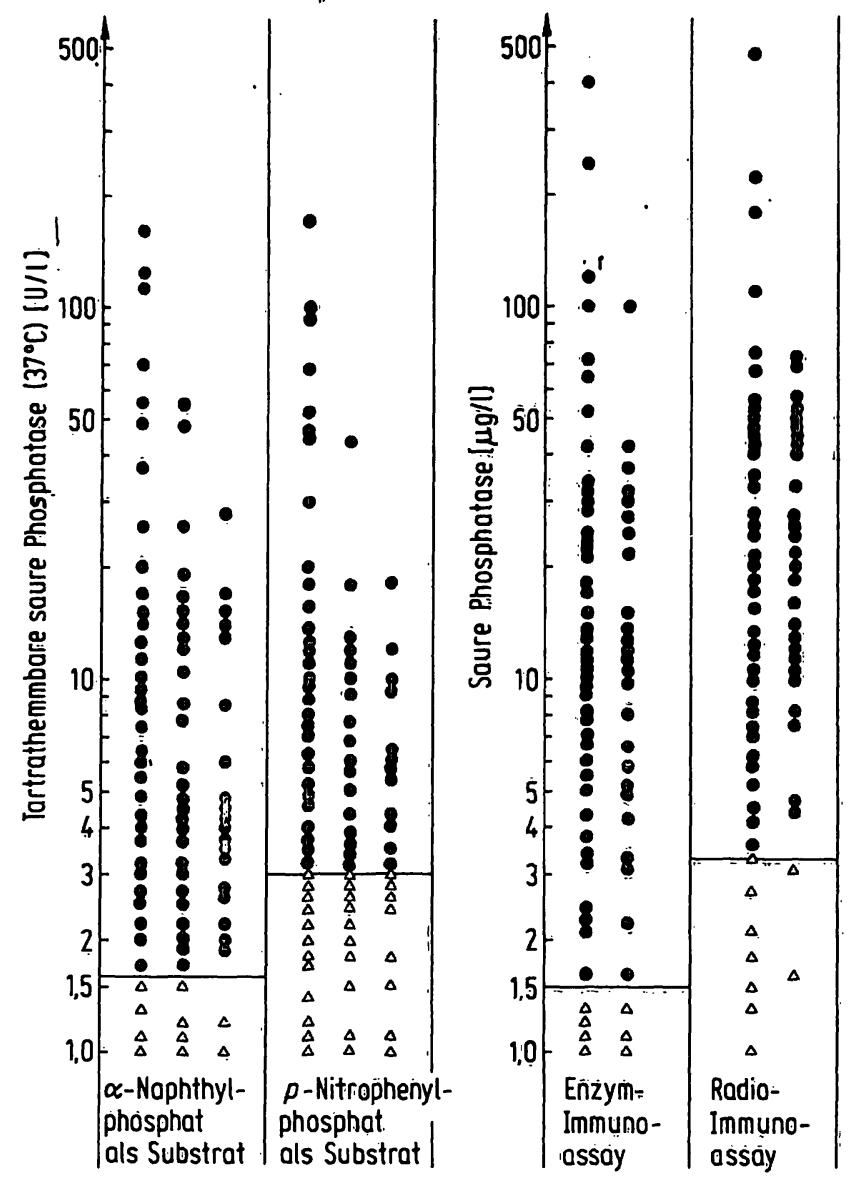

Abb. 2. Verteilung der Meßwerte an saurer Phosphatase bei Patienten mit Prostatacarcinomen der Stadien $C$ und $D$. Dargestellt sind die katalytischen Konzentrationeñ (U/l) im Serum von 89 Patienten für die Substrate $\alpha$-Naphthylphosphat und $p$-Nitrophenylphosphat; im Serum von 74 Patienten wurden zusätžlich die Konzentrationen ( $\mu \mathrm{g} /$ 1) an Prostata-spezifischer Phosphatase mit einem Enzym-Immunoassay und einem Radio=Immunoassay be-

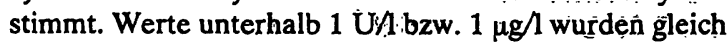
1 gesetzt.

o richtig positives Ergebnis

$\Delta$ falsch negatives Ergebnis.

Nimmt man den Meßwert der Obergrenze des Referenzbereiches als Trenngröße zwischen normal und pathologisch, dann lassen sich die Werte in richtig= positive und falsch-negative Ergebnisse aufteilen (9).

Die Anzahl an falsch-negativen Ergebnissen ist fürr das Substrat $p$-Nitrophenylphosphat am größten: 30 von 89 Patienten werden nicht als Carcinom-Patien= ten erkannt (Sensitivität des Tests: $66 \%$ ). Die übrigen Methoden erreichen eine wesentlich bèssère Sensitivität: Methode mit $\alpha$-Naphthylphosphat: $89 \%$, Enzym-Immunoassay $91 \%$ und Radio-Immunoassay $88 \%$. Tabelle 6 verdeutlicht, daß sich die Methode mit $\alpha$-Naphthylphosphat gegenüber den beiden immunchemischen Methóden nicht unterscheidet. Gegenüber der Methode mit $p$-Nitrophenylphosphat besteht jedoch ein signifikanter Unter- 
Tab. 6. Vergleich verschiedener Methoden zur Bestimmung der sauren Phosphatase: Vierfeldertafeln für Patienten mit ProstataCarcinom.

Die Meßwerte wurden mit dem jeweiligen Referenzwert der Methode verglichen und das Ergebnis gegenüber der Methode mit $\alpha$-Naphthylphosphat bewertet (falsch negativ: - ; richtig positiv: + ). Es bedeuten $s=$ signifikanter Unterschied bzw. $n s=$ nicht signifikanter Unterschied gegenüber der Methode mit $\alpha$-Naphthylphosphat (McNemar-Test, $\alpha=0,05$ ).

Referenzwerte: Tartrathemmbare saure Phosphatase: bis 1,6 U/1 (Substrat: $\alpha$-Naphthylphosphat) bzw. 3,0 U/l (Substrat: $p$ Nitrophenylphosphat); Enzym-Immunoassay: bis 1,5 $\mathrm{g} / \mathrm{l}$; Radio-Immunoassay: bis $3,3 \mu \mathrm{g} / \mathrm{l}$.

\begin{tabular}{|c|c|c|c|c|c|c|c|c|c|c|}
\hline & & \multicolumn{3}{|c|}{$\begin{array}{l}\text { Tartrathemmbare } \\
\text { saure Phosphatase } \\
p \text {-Nitrophenylphosphat }\end{array}$} & \multicolumn{3}{|c|}{ Enzymimmunoassay } & \multicolumn{3}{|c|}{ Radioimmunoassay } \\
\hline & & - & + & Total & - & + & Total & - & + & Total \\
\hline Tartrathemmbare saure Phosphatase & - & 10 & 0 & 10 & 6 & 2 & 8 & 7 & 1 & 8 \\
\hline \multirow[t]{2}{*}{$\alpha$-Naphthylphosphat } & + & 20 & 59 & 79 & 1 & 65 & 66 & 2 & 64 & 66 \\
\hline & Total & 30 & 59 & 89 & 7 & 67 & 74 & 9 & 65 & 74 \\
\hline Signifikanz & & & s & & & ns & & & ns & \\
\hline
\end{tabular}

schied. Wir meinen deshalb, daß die Methode mit $p$ Nitrophenylphosphat wegen ihrer unbefriedigenden Sensitivität aufgegeben werden sollte. Dabei haben wir die obere „Normgrenze“ der tartrathemmbaren sauren Phosphatase nicht auf 4 U/l gesetzt (10-12), sondern auf $3 \mathrm{U} / \mathrm{l}$ festgelegt. Dieser Wert entspricht dem Bedürfnis nach einer wünschenswerten besseren Sensitivität, an der Negativ-Einschätzung dieser Methode ändert sich jedoch nichts.

Es bleibt zu bedenken, daß immunchemische Methoden wegen der geringeren Störanfälligkeit bei der
Probenvorbereitung der hier vorgestellten Methode überlegen sein dürften. Inwieweit der Mehraufwand. an Geld, Zeit, Personal und Geräten, vor allem wegen der insgesamt doch niedrigen Sensitivität aller Tests in den Frühstufen des Prostata-Carcinoms, gerechtfertigt ist, muß vorerst offenbleiben. Nach unserer Meinung sollten die mit $\alpha$-Naphthylphosphat gemessenen katalytischen Konzentrationen an tartrathemmbarer saurer Phosphatase zwischen 1,6 und 2,0 U/1 (95. Perzentil bzw. Maximum) mit einer immunchemischen Methode kontrolliert werden.

\section{Literatur}

1. Hillmann, G. (1971) Z. Klin. Chem. Klin. Biochem. 9, 273274.

2. Bauer, H.-W., Walther, V. \& Schmiedt, F. (1980) Fortschr. Med. 98, 1301-1305.

3. Fishman, W. H. \& Lemer, F. (1953) J. Biol. Chem. 200, 8997.

4. Grenner, G. \& Schmidtberger, R. (1979) J. Clin. Chem. Clin. Biochem. 17, 156.

5. Foti, A. G., Herschman, H. \& Cooper, J. F. (1975) Cancer Res. 35, 2446-2452.

6. Hoffmann, G. E. \& Weiss, L. (1983) J. Clin. Chem. Clin. Biochem. 21, $31=33$.

7. Brühl, P. (1979) Diagnostik und Verlaufskontrolle maligner Tumoren des Urogenitaltraktes, Georg Thieme Verlag, D7000 Stuttgart 1.

8. Conover, W. J. (1971) Practical nonparametric statistics, J. Wiley \& Sons, New York.

9. Galen, R. S. \& Gambino, S. R. (1975) Beyond Normality, J. Wiley \& Sons, New York.

10. Griffiths, J. C. (1980) Clin. Chem. 26, 433-436. .

11. Cooper, E. H., Bauer, H.-W. \& Dati, F. (1982) Lab. Blätter 32, 87-107.

12. Bauer, H.-W. (1982) Med. Welt 33, 1616-1621.

Dr. rer. nat. W. Tritschler Boehringer Mannheim GmbH Erprobung Diagnostica Sandhofer Straße 116

D-6800 Mannheim 31

Prof. Dr. Dr. D. Seiler Städt. Krankenanstalten Institut für klinische Chemie Bremserstraße 79 D-6700 Ludwigshafen/Rh. 
